\title{
Convergence Acceleration for the Euler Equations Using a Parallel Semi-Toeplitz Preconditioner
}

\author{
Andreas Kähäri and Samuel Sundberg \\ Information Technology, Department of Scientific Computing, \\ Uppsala University, \\ Box 120, SE-751 04 Uppsala, Sweden, \\ Andreas.Kahari@tdb.uu.se, Samuel.Sundberg@tdb.uu.se \\ http://www.tdb.uu.se/
}

\begin{abstract}
We have studied a preconditioning technique for Krylov subspace methods on a fluid dynamics problem in 2-D. By discretizing the time-dependent Euler equations with a finite volume method in space and using the trapezoidal rule in time, we get a nonlinear system which is solved using a Newton-Krylov method. We precondition the linear iterates using a parallel semi-Toeplitz preconditioner to reduce the number of iterations. The experiments show a substantial reduction in the number of iterations required for convergence.
\end{abstract}

\section{Introduction}

We have studied a semi-Toeplitz preconditioner that has been extended by Domain Decomposition for use in parallel computations 3]. The use of semiToeplitz preconditioners for Krylov subspace methods has been thoroughly investigated by Hemmingsson [1, 2, 4] and have been found very efficient. Therefore it is of interest to parallelize them in an efficient way in order to use them in large scale computations.

This paper is focused on the convergence behavior of a Newton-Krylov method for a 2-D flowproblem. We first show how the global preconditioner system is solved, then continue by sketching the application and the discretization, and finally we present results.

\section{The Preconditioner}

By using Domain Decomposition we obtain a partitioned preconditioner system where we distinguish between interior unknowns $x^{I}$, boundary unknowns $x^{B, \bar{k}}$ and $x^{B, \bar{j}}$, and corner unknowns $x^{C}$, see Fig. 1. With these definitions the global preconditioner system $M x=y$ reads

P. Amestoy et al. (Eds.): Euro-Par'99, LNCS 1685, pp. 1124-1127 1999.

(C) Springer-Verlag Berlin Heidelberg 1999 

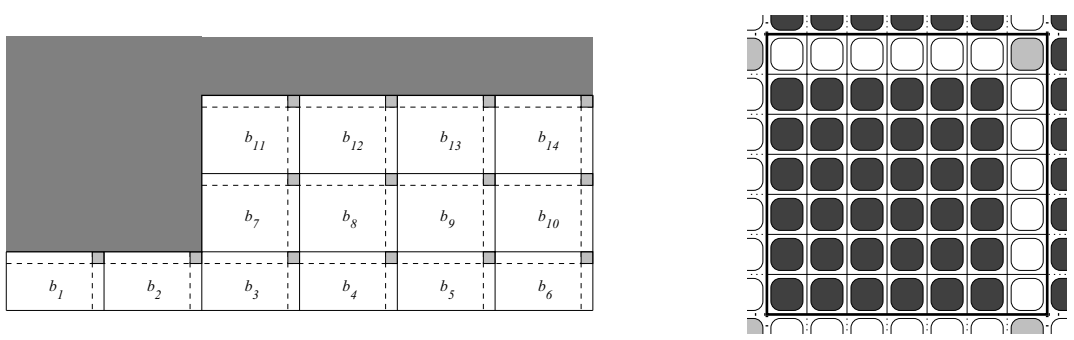

Fig. 1. The partitioning of the computational domain is shown in the picture to the left, and the right picture shows one of these blocks. Corner cells are grey, boundary cells are white and interior cells are black.

$$
\left[\begin{array}{cccc}
M^{I} & M^{I B, \bar{k}} & M^{I B, \bar{\jmath}} & 0 \\
M^{B I, \bar{k}} & M^{B, \bar{k}} & 0 & M^{B C, \bar{k}} \\
M^{B I, \bar{\jmath}} & 0 & M^{B, \bar{\jmath}} & M^{B C, \bar{\jmath}} \\
0 & M^{C B, \bar{k}} & M^{C B, \bar{\jmath}} & M^{C}
\end{array}\right]\left[\begin{array}{c}
x^{I} \\
x^{B, \bar{k}} \\
x^{B, \bar{\jmath}} \\
x^{C}
\end{array}\right]=\left[\begin{array}{c}
y^{I} \\
y^{B, \bar{k}} \\
y^{B, \bar{\jmath}} \\
y^{C}
\end{array}\right] .
$$

\subsection{Solution of the Preconditioner System}

We use the following solution strategy: (Step 1) solve for $x^{C}$, (Step 2) insert $x^{C}$ in (2.1) and solve for $x^{B, \bar{k}}$ and $x^{B, \bar{\jmath}}$ and (Step 3) insert $x^{B, \bar{k}}$ and $x^{B, \bar{\jmath}}$ in (2.1) and solve for $x^{I}$.

Interior unknowns If $x^{B, \bar{k}}$ and $x^{B, \bar{j}}$ are known, the solution for the interior unknowns decouples into the solution of as many independent systems of equations as there are subdomains in our computational domain. For a block $\ell$ we obtain the following system of equations

$$
M_{\ell}^{I} x_{\ell}^{I}=y_{\ell}^{I}-M_{\ell}^{I B, \bar{k}} x_{\ell}^{B, \bar{k}}-M_{\ell}^{I B, \bar{j}} x_{\ell}^{B, \bar{\jmath}}
$$

Since $M_{\ell}^{I}$ is defined such that it has a semi-Toeplitz structure, (2.2) is solved by means of modified sine transforms [2].

Block boundary unknowns For the boundary unknowns we start by eliminating the corner unknowns in (2.1). The resulting system of equations decouples into two independent systems of equations by, first assuming that $M^{I B, \bar{j}}$ is small and next assuming that $M^{I B, \bar{k}}$ is small, yielding the corresponding system for $x^{B, \bar{k}}$ and $x^{B, \bar{\jmath}}$. By block Gaussian elimination we get

$$
C^{B, \bullet} x^{B, \bullet}=y^{B, \bullet}-M^{B C, \bullet} x^{C}-M^{B I, \bullet}\left(M^{I}\right)^{-1} y^{I},
$$

where $C^{B, \bullet} \equiv M^{B, \bullet}-M^{B I, \bullet}\left(M^{I}\right)^{-1} M^{I B, \bullet}$.

The systems defined by (2.3), the so-called block boundary Schur complement systems, decouple into as many independent systems of equations as there are rows and columns of subdomains respectively. These are solved using modified sine transforms and the solution of narrow-banded systems. 
Corner unknowns Finally, we study the solution for the corner unknowns. The system for these unknowns is difficult to solve, and we have to make several approximations to obtain a system of equations that is easy to solve.

We start by making the approximation $M^{B I, \bullet} \approx 0$, which gives a system of equations that is decoupled from the interior unknowns. Using block Gaussian elimination we obtain

$$
C^{C} x^{C}=y^{C}-M^{C B, \bar{k}}\left(M^{B, \bar{k}}\right)^{-1} y^{B, \bar{k}}-M^{C B, \bar{\jmath}}\left(M^{B, \bar{\jmath}}\right)^{-1} y^{B, \bar{\jmath}},
$$

where $C^{C} \equiv M^{C}-M^{C B, \bar{k}}\left(M^{B, \bar{k}}\right)^{-1} M^{B C, \bar{k}}-M^{C B, \bar{\jmath}}\left(M^{B, \bar{\jmath}}\right)^{-1} M^{B C, \bar{\jmath}}$. We solve this system using a direct method.

\section{Model Problem}

We have studied the Euler equations on a backwards-facing step, where we use Crank-Nicholson for the time discretization. Spatial discretization is done by means of a finite volume method on a uniform grid. We let $(k, j)$ denote the center of a cell. In each cell $(k, j)$ with area $S_{k, j}$ our method is defined by

$$
\begin{aligned}
S_{k, j} \frac{q^{n+1}-q^{n}}{\Delta t} & + \\
& \sum_{\ell=1}^{4}\left[\theta\left(f n_{x}+g n_{y}\right)^{n+1}+(1-\theta)\left(f n_{x}+g n_{y}\right)^{n}\right] \Delta S_{\ell}=0,
\end{aligned}
$$

where the $\Delta S_{\ell}$ 's are the walls of the cell. The fluxes are computed using third order upwind approximations.

\subsection{Newton-Krylov Method}

The discretization in (3.1) above gives us a nonlinear system, $F\left(q^{n+1}\right)=0$, to solve in each time-step. We utilize Newton's method to solve this system, thus solving

$$
\nabla F\left(q^{n+1, \mu}\right) \Delta q^{n+1, \mu}=-F\left(q^{n+1, \mu}\right)
$$

and then adding

$$
q^{n+1, \mu+1}=q^{n+1, \mu}+\Delta q^{n+1, \mu} .
$$

We choose $q^{n+1,0}=2 q^{n}-q^{n-1}$ as initial guess, and let $q^{n+1}=q^{n+1, \mu+1}$ at convergence.

The linear system in 3.2 is solved using GMRES(6) where we use left preconditioning,

$$
M^{-1} \nabla F \Delta q=-M^{-1} F,
$$

to achieve faster convergence [5]. Here $M$ is the semi-Toeplitz preconditioner described above. 


\section{Results}

To study the performance of the preconditioner a series of experiments was run on a SMP cluster with three Digital Alpha servers, each hosting four Alpha EV5 processors. By taking 20 time-steps for different grid sizes, we found that the

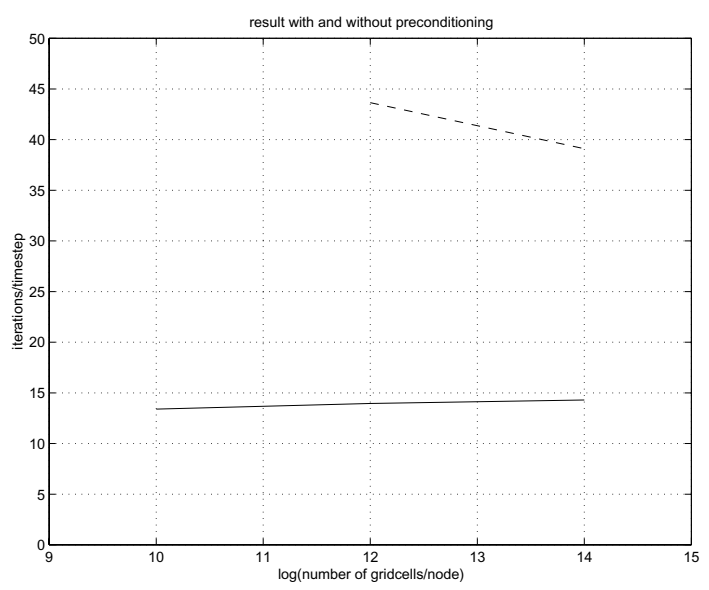

Fig. 2. Number of iterations/time-step with preconditioned (solid) and unpreconditioned (dashed) GMRES(6).

number of iterations per time-step was substantially reduced for the preconditioned method compared to the unpreconditioned method. Furthermore we found that the number of iterations was constant as the grid was refined, as shown in Fig. 2.

\section{References}

[1] L. Hemmingsson, A fast modified sine transform for solving block-tridiagonal systems with Toeplitz blocks, Numer. Algorithms, 7 (1994), pp. 375-389.

[2] — Toeplitz preconditioners with block structure for first-order PDEs, Numer. Linear Algebra Appl., 3 (1996), pp. 21-44.

[3] L. Hemmingsson And A. KäHÄRI, A new parallel preconditioner for the Euler equations, in Applied Parallel Computing. Large Scale Scientific and Industrial Problems, B. Kågström, J. Dongarra, E. Elmroth, and J. Waśniewski, eds., vol. 1541 of Lecture Notes in Comput. Sci., Springer-Verlag, Berlin, 1998, pp. 230-238.

[4] L. Hemmingsson And K. Отto, Analysis of semi-Toeplitz preconditioners for first-order PDEs, SIAM J. Sci. Comput., 17 (1996), pp. 47-64.

[5] Y. SAAd AND M. H. Schultz, GMRES: A generalized minimal residual algorithm for solving nonsymmetric linear systems, SIAM J. Sci. Statist. Comput., 7 (1986), pp. 856-869. 